\title{
Congenital Hypoplastic Trigeminal Nerve Revealed by Manifestation of Corneal Disorders Likely Caused by Neural Factor Deficiency
}

\author{
Naoyuki Morishige Yukiko Morita Naoyuki Yamada Teruo Nishida \\ Koh-Hei Sonoda
}

Department of Ophthalmology, Yamaguchi University Graduate School of Medicine, Ube, Japan

\section{Key Words}

Neurotrophic keratopathy · Trigeminal nerve $\cdot$ Corneal epithelial disorder

\begin{abstract}
Purpose: To report a case of hypoplastic trigeminal nerve associated with corneal epithelial disorders that were successfully treated with peptides derived from substance $P$ and insulinlike growth factor-1 (IGF-1). Case Report: A 16-month-old boy was referred for treatment of a persistent corneal epithelial defect on his left eye. Magnetic resonance imaging revealed the apparent absence of the trigeminal nerve on the left side, and the patient was therefore diagnosed with neurotrophic keratopathy. Treatment with eye drops containing the tetrapeptides FGLM-NH and SSSR derived from the neuropeptide substance $\mathrm{P}$ and the growth factor IGF-1, respectively, resulted in resurfacing of the corneal epithelial defect. Discussion: The trigeminal nerve anomaly of the patient likely gave rise to neurotrophic keratopathy as a result of a deficiency of neural factors, emphasizing the importance of neural regulation in corneal epithelial homeostasis.

(C) 2014 S. Karger AG, Basel
\end{abstract}

\section{Case Report}

A 16-month-old boy was referred to the Yamaguchi University Hospital on 10 December 2009 because of untreatable corneal opacity. He developed corneal opacity in his left eye at 2 months of age and was referred to the University Hospital, where he was diagnosed with infectious keratitis and treated with antibiotic eye drops. However, corneal opacity

Naoyuki Morishige, $\mathrm{MD}$ PhD

Department of Ophthalmology

Yamaguchi University Graduate School of Medicine

1-1-1 Minami-Kogushi, Ube, Yamaguchi 755-8505 (Japan)

E-Mail morishig@yamaguchi-u.ac.jp 
Morishige et al:: Congenital Hypoplastic Trigeminal Nerve Revealed by Manifestation of Corneal Disorders Likely Caused by Neural Factor Deficiency

remained although infectious keratitis was healed, and the boy was subsequently diagnosed with neurotrophic keratopathy and treated with autologous fibronectin eye drops and autologous serum eye drops. His mother reported the child's habit of rubbing his left eye with his hands.

On his first visit to our corneal service, we found ciliary injection, neovascularization, stromal opacity, and a central corneal epithelial defect in the left eye (fig. 1a, b). On the basis of his frequent blinking, we measured corneal sensation in the left eye of the patient with a Cochet-Bonnet esthesiometer and found it to be only $<5 \mathrm{~mm}$, whereas that in the right eye was normal $(55 \mathrm{~mm})$. Tear secretion was normal in both eyes. We performed cranial magnetic resonance imaging (MRI) to look for possible intracranial lesions. Such examination failed to reveal the left trigeminal nerve, although the right nerve appeared normal (fig. 2). Based on his selective touching of the left eye and the loss of left corneal sensation as well as the MRI findings, we diagnosed his condition as neurotrophic keratopathy of the left eye due to congenital hypoplastic trigeminal nerve. After obtaining written informed consent from his parents, we started to treat his left eye four times a day with eye drops containing the tetrapeptides FGLM- $\mathrm{NH}_{2}$ and SSSR with continuing levofloxacin eye drops for the prevention of secondary infectous keratitis during healing. After 5 days, the persistent epithelial defect was no longer apparent and the extent of stromal opacity was decreased in the left eye (fig. 1c, d).

\section{Discussion}

Clinical examination including MRI of the proband suggested that congenital hypoplastic trigeminal nerve and the consequent loss of neural regulation of the corneal epithelium were responsible for the severe corneal epithelial disorders. Congenital hypoplastic trigeminal nerve is a very rare clinical condition. Rosenberg [1] reviewed 43 cases and classified them into three groups. This particular patient was classified into group 1, in which isolated congenital anaesthesia occurred without any associated systemic or other neurological anomalies. As Ramaesh et al. [2] reviewed, several treatment strategies for congenital corneal anaesthesia were proposed for maintaining good visual acuity. Administration of eye drops containing nerve growth factor (NGF) was previously shown to be effective for the treatment of severe neurotrophic keratopathy in a child [3]. The patient in this previous study manifested reduced corneal sensitivity, but a trigeminal nerve abnormality was not detected by computed tomography or MRI of the brain or brain stem. Thus, the mechanism for the therapeutic action of NGF was not clear. It is possible that NGF promoted the expansion of corneal nerve fibers and the consequent provision of neural regulatory factors. It is also possible that NGF directly promoted the migration of corneal epithelial cells. We treated the present patient by topical administration of the peptides FGLM-NH $\mathrm{N}_{2}$ and SSSR. FGLM-NH $\mathrm{N}_{2}$ and SSSR are the minimal essential amino acid sequences derived from the neuropeptide substance P and insulin-like growth factor-1 (IGF-1) that mimic the synergistic stimulatory effect of the parent molecules on corneal epithelial migration [4, 5]. This combination of peptides has also previously proved effective for the treatment of persistent corneal epithelial defects in individuals with neurotrophic keratopathy [6]. The MRI finding of hypoplastic trigeminal nerve in the present case suggested that the affected cornea might be deficient in neural factors such as substance $P$, and the corneal epithelial disorders including reduced corneal sensitivity were indicative of neurotrophic keratopathy.

Functional and morphological examinations are required to provide insight into the pathogenesis of neurotrophic keratopathy. Functional examination includes evaluation of 
Morishige et al.: Congenital Hypoplastic Trigeminal Nerve Revealed by Manifestation of Corneal Disorders Likely Caused by Neural Factor Deficiency

corneal sensation with a Cochet-Bonnet esthesiometer, whereas corneal confocal microscopy is a key tool for morphological examination. We have previously applied corneal confocal microscopy to observe corneal nerve fibers in patients with neurotrophic keratopathy and have demonstrated reinnervation after treatment [7]. Given that the present patient was an infant, however, we were not able to examine his left eye by confocal microscopy.

In conclusion, we diagnosed hypoplastic trigeminal nerve in a child by MRI based on corneal findings. We have identified only one other case of a Japanese child with a hypoplastic trigeminal nerve revealed by autopsy. The incidence of this condition is not known but is thought to be low. It is likely that other symptoms of trigeminal palsy, such as loss of facial sensation and hypolacrimation, will become manifest in the present patient. However, his early diagnosis will allow the instigation of an appropriate treatment for any symptoms that could develop.

\section{Disclosure Statement}

T.N. owns a patent for FGLM-NH 2 plus SSSR. The other authors have no conflicts of interest to declare.

\section{References}

1 Rosenberg ML: Congenital trigeminal anaesthesia. A review and classification. Brain 1984;107(Pt 4):10731082.

-2 Ramaesh K, Stokes J, Henry E, Dutton GN, Dhillon B: Congenital corneal anesthesia. Surv Ophthalmol 2007;52:50-60.

-3 Tan MH, Bryars J, Moore J: Use of nerve growth factor to treat congenital neurotrophic corneal ulceration. Cornea 2006;25:352-355.

4 Nakamura M, Chikama T, Nishida T: Synergistic effect with Phe-Gly-Leu-Met-NH2 of the C-terminal of substance $\mathrm{P}$ and insulin-like growth factor-1 on epithelial wound healing of rabbit cornea. Br J Pharmacol 1999;127:489-497.

5 Yamada N, Yanai R, Kawamoto K, Nagano T, Nakamura M, et al: Promotion of corneal epithelial wound healing by a tetrapeptide (SSSR) derived from IGF-1. Invest Ophthalmol Vis Sci 2006;47:3286-3292.

-6 Yamada N, Matsuda R, Morishige N, Yanai R, Chikama TI, et al: Open clinical study of eye-drops containing tetrapeptides derived from substance $P$ and insulin-like growth factor- 1 for treatment of persistent corneal epithelial defects associated with neurotrophic keratopathy. Br J Ophthalmol 2008;92:896-900.

7 Morishige N, Komatsubara T, Chikama T, Nishida T: Direct observation of corneal nerve fibres in neurotrophic keratopathy by confocal biomicroscopy. Lancet 1999;354:1613-1614. 


\section{Case Reports in \\ Ophthalmology}

\begin{tabular}{l|l}
\hline Case Rep Ophthalmol 2014;5:181-185 \\
\hline DOI: 10.1159/000364899 & $\begin{array}{l}\text { ○ 2014 S. Karger AG, Basel } \\
\text { www.karger.com/cop }\end{array}$ \\
\hline
\end{tabular}

Morishige et al.: Congenital Hypoplastic Trigeminal Nerve Revealed by Manifestation of Corneal Disorders Likely Caused by Neural Factor Deficiency
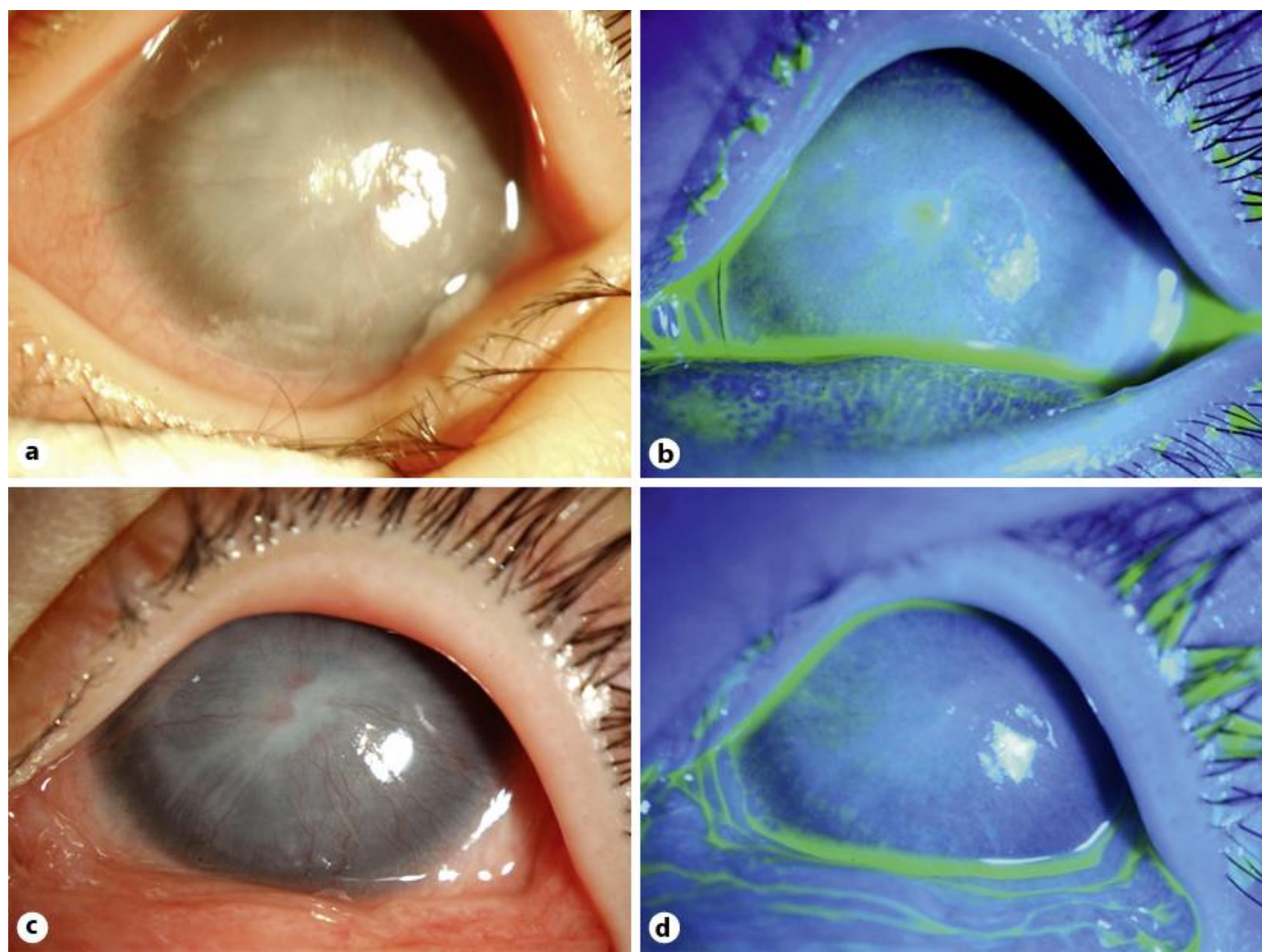

Fig. 1. Slitlamp photographs of the left eye of the 16-month-old male patient. a, b Diffuse corneal stromal opacity, neovascularization, and conjunctival injection were observed on his referral (a), and fluorescein staining also revealed an epithelial defect in the center of the cornea (b). c, d Five days after the administration of eye drops containing FGLM-NH 2 plus SSSR, the corneal stromal opacity and conjunctival injection were diminished (c) and the epithelial defect was no longer detectable by fluorescein staining (d). 
Case Reports in

Ophthalmology
Case Rep Ophthalmol 2014;5:181-185

DOI: $10.1159 / 000364899$

Morishige et al.: Congenital Hypoplastic Trigeminal Nerve Revealed by Manifestation

of Corneal Disorders Likely Caused by Neural Factor Deficiency

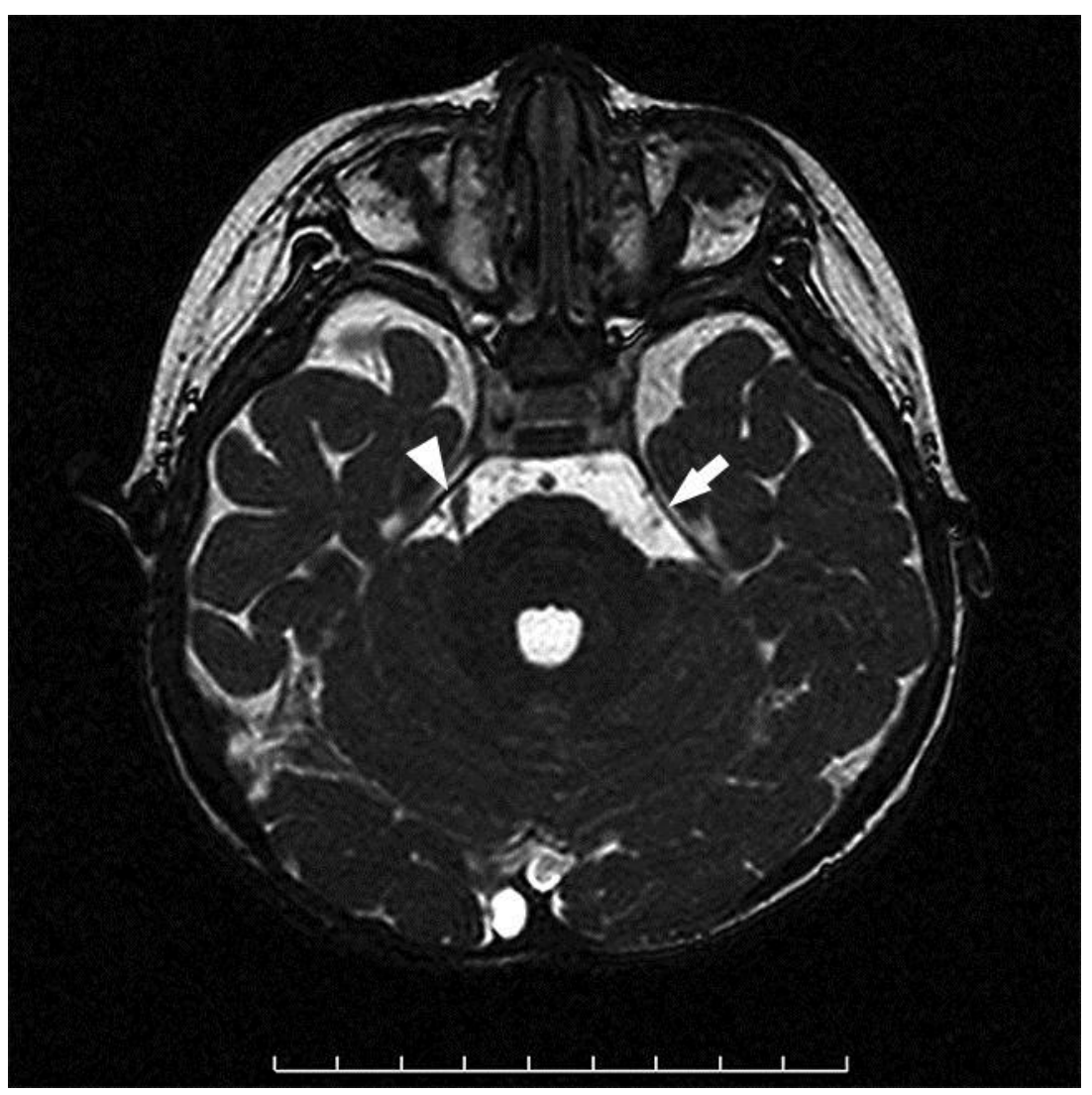

Fig. 2. MRI of the patient at the level of the brain stem. The trigeminal nerve was observed on the right side of the brain stem (arrowhead) but not on the left side (arrow). 